\title{
The MCDA technology and the Oil and gas industry: chronologic analysis
}

\author{
Carlos Eduardo Durange de Carvalho Infante
}

\begin{abstract}
A few years ago became notorious the inclusion of environmental and social issues in economic decisions, which led to a new strategic framework for organizations. Thus, it is of fundamental importance to know the present limitations and new paradigms in the operational and tactical developing of organizations, aiming at less striking and balanced actions. Thus, the objective was to develop an integrated interpretation of economic, environmental and social issues of the main companies in the oil and gas sectors in the world, featuring their activities through sustainability indicators covered in the three pillars of development (Triple Bottom Line). For the research robustness the decision support multicriteria analysis (AMD) was used as methodology, which in the application of the method ELECTRE III was possible to verify the ranking of companies according to direct sustainable reports. It was concluded that sustainable strategies allied to the Triple Bottom Line are a corporate and operational differential. Thus, it is expected to contribute to the deepening of enterprise policies across all strategic decisions focused on sustainability.

Keywords: Multicriteria analysis; Electre III; Oil and gas industry; Sustainability
\end{abstract}

\section{Introduction}

Across the globe, companies are faced with the responsibility not to cause damage to the environment, or at least minimize it. In industrialized nations, more companies are including sustainability in their business; they believe being capable of reducing pollution and increasing profits simultaneously (Hart, 1996). In third world countries, the demands for effective implementation of sustainability have also experienced considerable increase in face of the global view of economic development (Kumah, 2006).

In this context, the number of reports on sustainability performance of companies presented to stakeholders and shareholders has increased in the recent years. One of the key purposes of this report, according to the Global Reporting Initiative (GRI), is to allow a comparison among companies and a performance evaluation for every year (GRI, 2012). This article aims at checking for this possibility, through the use of reports for benchmarking and comparison. It focuses on the important case of the oil and gas industry worldwide, comparing the five largest companies in the period 2005e2011. It also analyzes the year-by-year evolution for each company.

The Multicriteria Decision Analysis (MCDA) was used to obtain the ranking of companies. The MCDA term refers to various methods developed to help decision makers achieve robust and promising results (Loken, 2007), and can be used in various areas, e.g., solid waste management (Karmperis et al., 2013) and assessment of biodiversity conservation (Bottero et al., 2013). Among the existing methods, the ELECTRE III was chosen for reasons that will be detailed in Section 3.

The paper is organized as follows. The first part consists of this introduction, Section 1, followed by detailing the data analyzed, Section 2. In Sections 3 and 4 there is the method description and thereupon the results. Finally, Section 5 shows the conclusion synthesizing the study and results.

\section{Data}

The top five companies in the oil and gas sector worldwide were analyzed. They account for over $50 \%$ of the world oil industry investment (Passuello et al., 2012).

These companies were compared by means of their sustainability reports, all prepared according to the GRI guidelines, version 3 (G3). Aggregate data from reports between 2005 and 2013 were used, i.e. in the nine years preceding the important Macondo accident in 2010. This accident caused major repercussions in the international media and directly impacted one of the selected companies. This company affected by Macondo accident was BP British Petroleum.

The GRI sustainability reports consist of two parts: general information and information on economic, environmental and social indicators. This article made use of the latter one. In G3, the total number of indicators is seventy-nine, but not all companies are obliged to report all of them.

For data selection and comparison of companies, the following steps were followed:

a) The five companies selected were defined according to their market value. Table 1 shows the five largest companies in the oil and gas sector, with their nomenclature and market value. 
The MCDA technology and the Oil and gas industry: chronologic analysis

Table1 - List of the selected companies

\begin{tabular}{|c|c|c|}
\hline Companies & Nomenclature & Market value (million USD - 2010) \\
\hline Exxon Mobil Coporation & E4 & USD 303,30 \\
\hline Royal Dutch Shell & E3 & USD 168,00 \\
\hline Petrobrás S.A & E1 & USD 147,80 \\
\hline Chevron Corporation & E5 & USD 147,20 \\
\hline British Petroleum - BP & E2 & USD 116,90 \\
\hline
\end{tabular}

The indicators of environmental performance, economic and social reports of all companies were collected and analyzed regarding the sustainable performance improvement. An important contribution can also be seen in Lang et al. (2007). It is noteworthy that as GRI signatories, they apply the GRI G3 Guidelines for preparing their sustainability reports.

It was sought to compare the evolution of these companies' activities over nine years, i.e. 2005-2013.

Frame 1 - Qualitative weights referring to the criterion EC8

\begin{tabular}{|c|c|}
\hline Scale & Impact on the companies' activities \\
\hline Very high - 5 & $100 \%$ ofcriterionapplication \\
\hline High - & $75 \%$ ofcriterionapplication \\
\hline Medium - 3 & $50 \%$ ofcriterionapplication \\
\hline Low - 2 & $25 \%$ ofcriterionapplication \\
\hline Verylow - 1 & $5 \%$ ofcriterionapplication \\
\hline
\end{tabular}

b) The indicators (Frame 2) were selected from the following criteria (Worrall et al., 2009):

i. Relevance to the sector under study;

ii. Contribution to the Triple Bottom Line analysis; and

iii. Reporting and full disclosure by all companies selected.

An important observation can be made for the criterion EC8. This criterion has a qualitative scale as standardization measure, since its weights assigned were given by the scale described in Frame 1.

Frame 2 - Description and relevance of criteria

\begin{tabular}{|c|c|}
\hline Criteria & RELEVANCE \\
\hline \multicolumn{2}{|l|}{ Economic } \\
\hline EC1 - Total production & $\begin{array}{l}\text { Data on the creation and distribution of economic value provide a basic indication of } \\
\text { how the organization } \\
\text { has created wealth for stakeholders. }\end{array}$ \\
\hline $\begin{array}{l}\text { EC8 - Development and } \\
\text { impact of investments } \\
\text { in infrastructure and } \\
\text { services }\end{array}$ & $\begin{array}{l}\text { The impacts on investments in infrastructure can go beyond the scope of their own } \\
\text { organization's } \\
\text { business operations and achieve a longer time scale. Thismay include transport } \\
\text { connections, publicservices, etc }\end{array}$ \\
\hline \multicolumn{2}{|l|}{ Environmental } \\
\hline $\begin{array}{l}\text { EN3 - Direct energy } \\
\text { consumption is criminated } \\
\text { by primary energy source }\end{array}$ & $\begin{array}{l}\text { The organization's ability to efficiently use the energy can be revealed by means of } \\
\text { calculating the amount of energy it consumes. Energy } \\
\text { consumptionhasdirecteffectonoperatingcosts. }\end{array}$ \\
\hline $\begin{array}{l}\text { EN8 - Total water withdrawal by } \\
\text { source. }\end{array}$ & $\begin{array}{l}\text { The disclosure of the total water withdrawal by source contributes to the } \\
\text { understanding of the overall magnitude of potential impacts and risks associated with } \\
\text { the water use by the organization. }\end{array}$ \\
\hline $\begin{array}{l}\text { EN16 - Total direct emissions of } \\
\text { greenhouse gases per weight. }\end{array}$ & $\begin{array}{l}\text { Emissions of greenhouse gases are the main cause of climate change. Direct emission } \\
\text { is all emissions from sources owned or in the possession of the company. }\end{array}$ \\
\hline $\begin{array}{l}\text { EN50 - Total indirect } \\
\text { emissions of greenhouse } \\
\text { gases per weight. }\end{array}$ & $\begin{array}{l}\text { In some organizations, the indirect emissions of greenhouse gases are higher than } \\
\text { direct emissions. } \\
\text { The changes in their practices can reduce these emissions considerably. Indirect } \\
\text { emission is all emissions consequent of the company's activities. }\end{array}$ \\
\hline EN20 - SOx, by type and weight & $\begin{array}{l}\text { Measures the magnitude of organization's atmospheric emissions and can } \\
\text { demonstrate the size and importance } \\
\text { of these emissions compared to others. }\end{array}$ \\
\hline EN60 - NOx, by type and weight & Air pollutants cause adverse effects on habitats and on human and animal health. \\
\hline $\begin{array}{l}\text { EN21 - Total water discharge by } \\
\text { quality and destination. }\end{array}$ & $\begin{array}{l}\text { The volume and quality of water discharged (wasted) by the reporting organization } \\
\text { are directly linked to environmental impact and operating costs. }\end{array}$ \\
\hline EN22 - Waste total weight & $\begin{array}{l}\text { Data on waste generation over the years may indicate the level of progress that the } \\
\text { organization has achieved in the } \\
\text { effort to reduce waste. }\end{array}$ \\
\hline $\begin{array}{l}\text { EN23 - Total volume of } \\
\text { significant spills. }\end{array}$ & $\begin{array}{l}\text { Accidental spills of chemicals, oils and fuels can have significant negative impacts } \\
\text { on the environment, potentially affecting soil, water, air, biodiversity } \\
\text { and human health. }\end{array}$ \\
\hline $\begin{array}{l}\text { EN30- Total investments } \\
\text { and expenditures in environmental } \\
\text { protection }\end{array}$ & $\begin{array}{l}\text { The measurement of environmental mitigation and environmental protection } \\
\text { expenditures allows organizations to assess the efficiency of their environmental } \\
\text { initiatives. It also provides valuable }\end{array}$ \\
\hline
\end{tabular}


The MCDA technology and the Oil and gas industry: chronologic analysis

\begin{tabular}{|l|l|}
\hline by type & data for cost/benefit internal analysis. \\
\hline SOCIAL & $\begin{array}{l}\text { The size of the workforce provides an overview of the extent of impacts generated by } \\
\text { labor issues. } \\
\text { LA1 - Total workforce by } \\
\text { conployment type, employment } \\
\text { and region. }\end{array}$ \\
\hline LA7 - Rates of work-related deaths & $\begin{array}{l}\text { The safety and health performance is a key measure of the duty of care to na } \\
\text { organization. }\end{array}$ \\
\hline $\begin{array}{l}\text { LA70 - Rates of work-related } \\
\text { occupational illnesses by region. }\end{array}$ & Health management practices that result in a number of minor incidents at work. \\
\hline
\end{tabular}

c) The standardization of measures for each criterion followed a logic that can be seen in Frame 3. The economic and environmental criteria were normalized to the amount produced, i.e. the total annual production, which encourages the company's economic expansion and establishes a magnitude comparison between them. The social criteria were normalized according to the total number of employees in the particular year, company, since these criteria are of major impact on life quality of workers and families.

Frame 3 - Criterianormalized

\begin{tabular}{|c|c|}
\hline Criteria & Normalization \\
\hline \multicolumn{2}{|l|}{ Economics } \\
\hline EC1 - Total production & $10^{3} \mathrm{oil} / \mathrm{day}$ \\
\hline $\begin{array}{l}\text { EC8 - Development and } \\
\text { impact of investments } \\
\text { in infrastructure and } \\
\text { services }\end{array}$ & Qualitativeweight \\
\hline \multicolumn{2}{|l|}{ Environmental } \\
\hline $\begin{array}{l}\text { EN3 - Direct energy } \\
\text { consumption is criminated } \\
\text { by primary energy source }\end{array}$ & TJ/barrels/year \\
\hline EN8 - Total water withdrawal by source. & $10^{3} \mathrm{~m}^{3} / 10^{3}$ barrels $/$ year \\
\hline EN16 - Total direct emissions of greenhouse gases per weight. & $\mathrm{Mt} / 10^{3}$ barrels/year \\
\hline $\begin{array}{l}\text { EN50 - Total indirect } \\
\text { emissions of greenhouse } \\
\text { gases per weight. }\end{array}$ & $\mathrm{Mt} / 10^{3}$ barrels/year \\
\hline EN20 - SOx, by type and weight & $\mathrm{t} / 10^{3}$ barrels/year \\
\hline EN60 - NOx, by type and weight & $\mathrm{t} / 10^{3}$ barrels/year \\
\hline EN21 - Total water discharge by quality and destination. & $103 \mathrm{~m}^{3} / 10^{3}$ barrels/year \\
\hline EN22 - Waste total weight & $\mathrm{t} / 10^{3}$ barrels/year \\
\hline $\begin{array}{l}\text { EN23 - Total volume of } \\
\text { significant spills. }\end{array}$ & $\mathrm{m} 3 / 10^{3}$ barrels/year \\
\hline $\begin{array}{l}\text { EN30- Total investments } \\
\text { and expenditures in environmental protection } \\
\text { by type }\end{array}$ & $10^{3} \mathrm{USD} / 10^{3}$ barrels/year \\
\hline \multicolumn{2}{|l|}{ Social } \\
\hline $\begin{array}{l}\text { LA1 - Total workforce by } \\
\text { employment type, employment contract } \\
\text { and region. }\end{array}$ & $10^{3}$ employees \\
\hline LA7 - Rates of work-related deaths & (A)/employees \\
\hline LA70 - Rates of work-related occupational illnesses by region. & rate $/ 10^{4}$ person hours \\
\hline
\end{tabular}

d) The purpose of each criterion can be observed in Frame 4. These objectives are of paramount importance for the correct application of the ELECTRE III method.

Frame 4 - Objective of each criterion

\begin{tabular}{|c|c|}
\hline Criteria & Objective \\
\hline EC1 & Maximize \\
\hline EC8 & Maximize \\
\hline EN3 & Minimize \\
\hline EN8 & Minimize \\
\hline EN16 & Minimize \\
\hline EN50 & Minimize \\
\hline EN20 & Minimize \\
\hline EN60 & Minimize \\
\hline EN21 & Minimize \\
\hline EN22 & Minimize \\
\hline EN23 & Minimize \\
\hline EN30 & Maximize \\
\hline LA1 & Maximize \\
\hline LA7 & Minimize \\
\hline LA70 & Minimize \\
\hline
\end{tabular}




\section{Method}

The multicriteria approaches propose ways to model the decision-making processes, including items such as type of decision to be made, unknown events that may affect the results, possible courses of action and the results themselves. The multicriteria are also used to measuring the sustainability (Tosicey al., 2015; Castellini et al., 2012) and others scientific areas. Among the most robust multicriteria methods, the specific methodology of ELECTRE Family stands out (Roy, 1985).

\subsection{ELECTRE III}

Within the ELECTRE family, the method chosen was ELECTRE III that allows the use of inaccurate, indefinite and uncertain criteria, inherent to complex processes in human decision, based on the use of pseudocriteria and thresholds of preference and indifference. Moreover, the "very bad" performance in one criterion that cannot be offset by good results in other criteria depending on the veto threshold. ELECTRE III has been widely used. In order to exemplify it, some practices are applied: in classification problems, for example, in the ranking of actions for investments selection (Huck, 2009), the choice for a strategic sustainable management of demolition waste (Roussat et al., 2009), energy systems selection (Tosic, et al., 2015; Cavallaro, 2010), housing evaluation (Natividade-Jesus et al., 2007), environment and management of water consumption (Mushtaq Khan, et al., 2015;Giner-Santonja et al., 2012; Hanandeh and El-Zein, 2010), finance (Zhelev, 2014; Li and Sun, 2010), decision analysis (Infante, et al., 2013; Montazer et al., 2009), education (Giannoulis and Ishizaka, 2010) and others (Bana e Costa and Oliveira, 2012; Durbach and Stewart, 2012; Frini et al., 2012). However, it has not been applied to the ordination and performance evolution analysis of the greatest oil and gas industries worldwide.

The ELECTRE III depends on the construction and exploitation of some relationships. Its phases are depicted in Fig. 1.

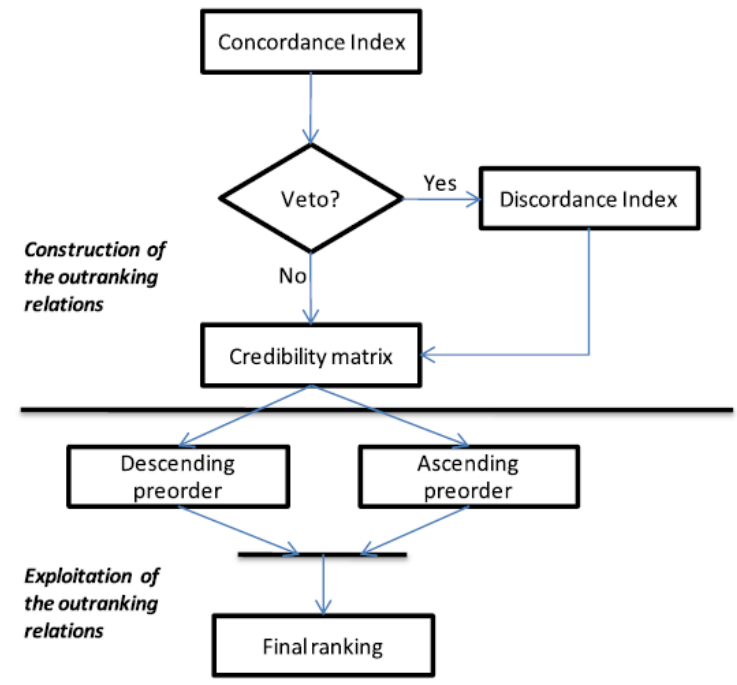

Fig. 1 - Electre III flow

- Construction of the outranking relationship: the performance alternatives (the five companies under study) are pairwise compared (A, B). Each pairwise is characterized by an overcome relationship. Establish that "the alternative A outperforms alternative B" means "alternative A is at least as good as alternative B". There are three overcome relationships: "indifferent," "weakly preferred" or "strictly preferred", according to the difference between the performance alternatives and thresholds given by the decision maker.

- Exploitation of the outranking relationship: two preclassifications are then constructed with two antagonist procedures (upward and downward distillation). The combination of the two pre-classifications provides the final classification.

\subsection{Constructing the outranking relationships}

\subsubsection{Pseudo-criteria}

The simplest and most traditional criterion is called 'true criteria'. These have no defined limits. Only the difference among criteria scores is used to determine which option is the preferred one. Pseudo-criteria are used in order to take into account the inaccuracy and uncertainty in indeterminacy in complex decision problems. The indifference $\mathrm{q}$ and preference $\mathrm{p}$ thresholds allow the construction of a pseudo-criterion. Thus, three alternative relationships between alternatives A and B can be considered: 
a) $\mathrm{A}$ and $\mathrm{B}$ are indifferent if the difference between the performance of two alternatives issmaller than the threshold indifference. The indifference between alternatives is denoted as A I B.

A I B if; and only if; $\mathrm{z}(\mathrm{A})-\mathrm{z}(\mathrm{B}) \leq \mathrm{q}$

where, $\mathrm{z}(\mathrm{X})$ : alternative $\mathrm{X}$ performance; $\mathrm{q}$ : indifference threshold.

b) Alternative $\mathrm{A}$ has weak preference compared to alternative $\mathrm{B}$ if the difference between their performances is between the thresholds of indifference and preference. The notation for weak preference is A $\mathbf{Q} B$.

A Q B if; and only if; $q<z(A)-z(B) \leq p$

where, $\mathrm{z}(\mathrm{X})$ : alternative $\mathrm{X}$ performance; $\mathrm{q}$ : indifference threshold; $\mathrm{p}$ : preference threshold.

c) Alternative $\mathrm{A}$ is strictly preferred to alternative $\mathrm{B}$ if the difference between the alternative performances is greater than the threshold preference. The notation is strictly preferential A $\mathbf{P}$ B.

A P B if; and only if; $z(A)-z(B) \geq p$

where, $\mathrm{z}(\mathrm{X})$ : alternative $\mathrm{X}$ performance; $\mathrm{p}$ : preference threshold.

\subsubsection{Concordance index}

The concordance index (Eqs. (4) and (5)) indicates the truth of the statement "alternative A outperforms alternative B" (A S B). C = 1 indicates the full truth of the assertion and C $=0$ indicates that the statement is false. The graphic representation is given in Fig. 2.

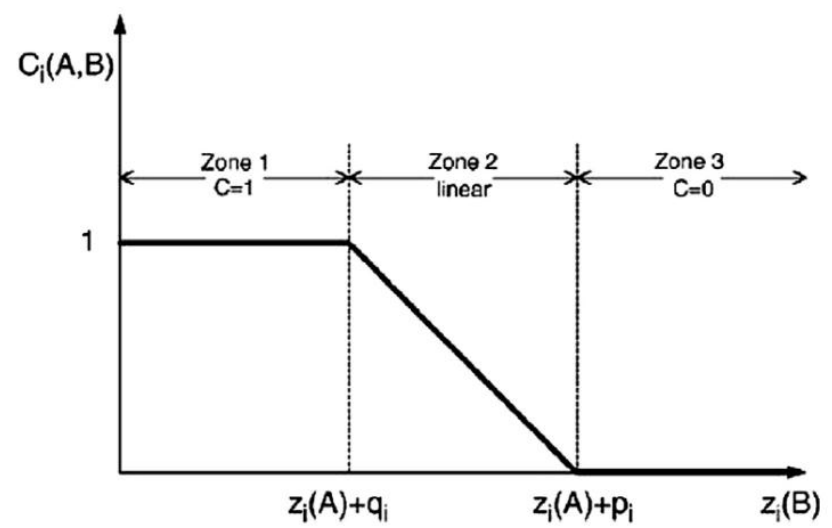

Fig.2 - Concordance index between A and B alternatives

Zone $1 . \mathrm{Z}_{\mathrm{i}}(\mathrm{B})-\mathrm{Z}_{\mathrm{i}}(\mathrm{A}) \leq \mathrm{q}_{\mathrm{i}}$, alternatives $\mathrm{A}$ and $\mathrm{B}$ are indifferent, which means agreementon the statement "The alternative Aovercomes alternative B".Zone 2. $\mathrm{q}_{\mathrm{i}}<\mathrm{z}_{\mathrm{i}}(\mathrm{B})-\mathrm{z}_{\mathrm{i}}(\mathrm{A})<\mathrm{p}_{\mathrm{i}}$, the alternative B is weakly preferred to A, which means a partial agreement on the statement "The alternative A overcomes the alternative B". Zone 3. $Z_{i}(B)-Z_{i}(A) \geq p_{i}$, alternative $B$ is strictly preferred to $A$, which means a false agreement on the statement "alternative A overcomes alternative B".

$C(a, b)=\frac{1}{k} \cdot \sum_{j=1}^{n} k j \cdot c j(a, b)$

Being for each criterion,

$$
c_{j}(a, b)=\left\{\begin{array}{c}
\text { if } g_{j}(a)+q_{j}(b) \geq g_{j}(b) \\
0 \text { if } g_{j}(a)+p_{j}(b) \leq g_{j}(b) \\
p_{j}+g_{j}(a)-g_{j}(b), \text { in all cases } \\
p_{j}-q_{j}
\end{array}\right.
$$

where, $\mathrm{C}(\mathrm{a}, \mathrm{b})$ : concordance index of actions a and $\mathrm{b} ; \mathrm{K}$ : sum of all weights of criteria; $\mathrm{k}_{\mathrm{j}}$ : weight of criterion $\mathrm{j}$, for $\mathrm{j}=1,2,3, \ldots, n ; \mathrm{c}_{\mathrm{j}}$ : concordance index of actions a and $\mathrm{b}$, under the criterion $\mathrm{j}$.

\subsubsection{Discordance index}

If the difference in performances between alternatives $\mathrm{A}$ and $\mathrm{B}$ in a criterion $\mathrm{i}$ is greater than the veto threshold $\mathrm{v}_{\mathrm{i}}$ it is cautious to refuse the statement "alternative A overcomes alternative B". The discordance index for each criterion $\mathrm{i}$ is given by Eq. (6). Fig 3 shows the graphic representation of this index. 


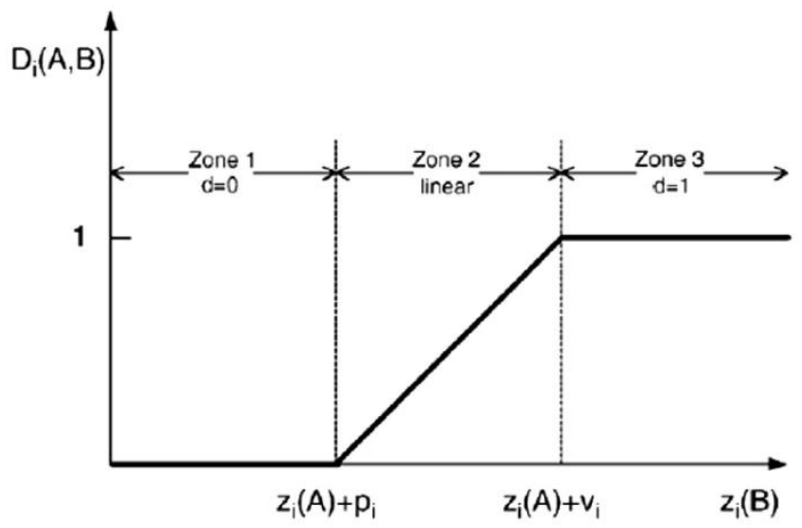

Fig.3 - Disagreement index between A and B alternatives

Zone $1 . \mathrm{Z}_{\mathrm{i}}(\mathrm{B})-\mathrm{Z}_{\mathrm{i}}(\mathrm{A}) \leq \mathrm{p}_{\mathrm{i}}$, alternative $\mathrm{B}$ is weakly preferable to alternative $\mathrm{A}$, which means no disagreement about the statement "alternative $A$ overcomes alternative $B$ ". Zone 2 . $P_{i}<z_{i}(B)-z_{i}(A)<v_{i}$, alternative $B$ is strictly preferred to alternative $A$, which means weak disagreement on the assertion "alternative A overcomes alternative $\mathrm{B}$ ". Zone $3 . \mathrm{Z}_{\mathrm{i}}(\mathrm{B})-\mathrm{Z}_{\mathrm{i}}(\mathrm{A}) \geq \mathrm{v}_{\mathrm{i}}$, the difference between alternative $\mathrm{A}$ and alternative $\mathrm{B}$ exceeds the threshold for veto, which means total disagreement with the statement "alternative A overcomes alternative B".

$$
\begin{gathered}
1 \text { se }_{j}(a)+v_{j} \leq g_{j}(b) \\
d_{j}(a, b)=
\end{gathered} \quad\left\{\begin{array}{l}
0 \text { se }_{j}(a)+p_{j} \geq g_{j}(b) \\
g_{j}(b)-g_{j}(a)-p_{j} \text { nos demais casos } \\
v_{j}-p_{j}
\end{array}\right.
$$

where: $\mathrm{z}_{\mathrm{i}}(\mathrm{X})$ : alternative $\mathrm{X}$ performance in criterion $\mathrm{i} ; \mathrm{p}_{\mathrm{i}}$ : threshold of alternative preference on the criterion $\mathrm{i}$.

\subsubsection{Credibility index}

Considering the concordance (Eq. (4)) and discordance (Eq. (6)) indexes, the credibility degree (Eq. (7)) indicates whether the outranking hypothesis is true or not. If the concordance index (Eq.(4)) is greater than or equal to the discordance index on all criteria (Eq. (6)), then Eq. (7) is equal to Eq. (4). If Eq. (4) is strictly below Eq. (6) then the reliability degree (Eq. (7)) is equal to Eq. (4). Note the importance of the direct relationship of these indices.

$$
\mathrm{S}(\mathrm{a}, \mathrm{b})=\left\{\begin{array}{r}
\mathrm{C}(\mathrm{a}, \mathrm{b}), \mathrm{se}_{\mathrm{j}}(\mathrm{a}, \mathrm{b}) \leq \mathrm{C}(\mathrm{a}, \mathrm{b}) \forall_{\mathrm{j}} \\
\mathrm{C}(\mathrm{a}, \mathrm{b}) . \prod_{j \in J(a, b)} \frac{1-d j(a, b)}{1-C(a, b)}, \text { otherwise }
\end{array}\right.
$$

Where: $\mathrm{J}(\mathrm{A}, \mathrm{B})$ : is the set of criteria for $\operatorname{di}(\mathrm{A}, \mathrm{B})>\mathrm{C}(\mathrm{A}, \mathrm{B})$.

\subsection{Performance Matrix}

\section{Results}

In order to determine the sequence of alternatives using the processes assigned to the ELECTRE III, the performance matrix (Tables 2, 3 and 4) of alternatives for each criterion can be observed taking into account the evolution over nine years. For each criterion in Tables 2, 3 and 4, thresholds and weights were assigned by experts through questionnaires and interviews conducted directly. Tables 5 and 6 show the values for each threshold (preference, indifference and veto). In the case of weights, all these criteria at this first time, receive the same importance in the analysis, i.e., equal weights were assigned to all of them $(\mathrm{kj} 1 / 41)$. After calculating the indices of concordance and disagreement, the degrees of credibility are built and consolidated in the Matrix of Credibility, Tables 7 and 8 . The degrees of credibility and indexed to each pair of alternatives do not produce a symmetric matrix. The next step is to explore this matrix. See Section 4.2. 
The MCDA technology and the Oil and gas industry: chronologic analysis

Table2 - Performance Matrix - 2005 - 2007

\begin{tabular}{|c|c|c|c|c|c|c|c|c|c|c|c|c|c|c|c|}
\hline \multirow[t]{3}{*}{ Criterla } & \multicolumn{15}{|l|}{ Alternatives } \\
\hline & \multicolumn{5}{|l|}{2005} & \multicolumn{5}{|l|}{2006} & \multicolumn{5}{|l|}{2007} \\
\hline & E1 & E2 & $E 3$ & $E 4$ & $E 5$ & E1 & E2 & E3 & $E 4$ & $E 5$ & E1 & E2 & E3 & $E 4$ & $E 5$ \\
\hline \multicolumn{16}{|l|}{ Economlc } \\
\hline $\mathrm{BCl}$ & 2,217 & 4,014 & 3,850 & 4,100 & 2,500 & 1,892 & 3,926 & 1,40 & 4,200 & 2,670 & 2,300 & 3,818 & 1,520 & 4,200 & 2,619 \\
\hline BCS & 5,000 & 4,000 & 3,000 & 5,000 & 3,000 & 5,000 & 4,000 & 2,000 & 5,000 & 3,000 & 5,000 & 4,000 & 3,000 & 5,000 & 3,000 \\
\hline \multicolumn{16}{|c|}{ Environmental } \\
\hline $\mathrm{EN} 3$ & 644,599 & 894,000 & 401,000 & 1004,343 & 985,205 & $811,7 \pi$ & 718,777 & 985,731 & $1014.351,000$ & 973,696 & 660,418 & 739,109 & 955,443 & 101,109 & $1.013,187$ \\
\hline EN8 & 195,871 & 325,573 & 454,012 & 76,846 & 304,658 & 258,913 & 238,662 & $1.065,49$ & 201,566 & 317,069 & 257,880 & 215,274 & 1034,607 & 210,698 & 300,230 \\
\hline EN16 & 57,217 & 49,962 & 74,720 & 92,215 & 65,425 & 72,200 & 41,382 & 186,454 & 94,912 & 63,516 & 59,547 & 45,566 & 165,826 & 91,977 & 67,055 \\
\hline EN50 & 3,868 & 9,487 & 5,693 & 14,166 & 16,219 & 0,825 & 7,048 & 11,796 & 7,175 & 0,636 & 0,429 & 7,678 & 9,012 & 5,871 & 0,837 \\
\hline $\mathrm{E} 220$ & 187,432 & 84,635 & 229,852 & 167,056 & 130,411 & 189,696 & 73,971 & 563,166 & 153,947 & 121,082 & 179,750 & 71,041 & 474,045 & 136,986 & 96,241 \\
\hline Ev60 & 275,684 & 148,794 & 130,938 & 106,916 & 133,699 & 338,122 & 136,777 & 342,466 & 105,023 & 141,604 & 265,396 & 146,387 & 308,219 & $\begin{array}{ll}97,847 \\
\end{array}$ & 151,684 \\
\hline EN21 & 184,131 & 88,906 & 331,614 & 334,113 & 241,096 & 236,468 & 119,331 & $1.054,033$ & 161,122 & 477,143 & 205,837 & 115,531 & 937,275 & 133,725 & 314,875 \\
\hline $\mathbb{E} 22$ & 657,435 & 161,763 & 315,958 & 198,463 & 283,836 & 456,564 & 188,417 & 300,609 & 160,470 & 227,797 & 352,591 & 121,989 & 484,859 & 109,589 & 206,081 \\
\hline EN23 & 332,425 & 300,319 & 180,751 & 200,468 & 282,740 & 424,281 & 244,245 & $1.198,630$ & 192,433 & 343,748 & 459,797 & 717,581 & 1207,642 & 164,384 & 347,304 \\
\hline $\mathrm{E} 30$ & $1.005 .783,746$ & $3.8222238,603$ & $9.037 .537,805$ & $2.205 .145,339$ & $3.791 .780,822$ & $924,192,864$ & $1.744 .603,940$ & $5.0988 .934,551$ & 20926288,832 & $892.719,717$ & $1.330 .107,038$ & $787.186,865$ & $5.046 .863,735$ & $2.478 .799,739$ & $1464.534,723$ \\
\hline \multicolumn{16}{|l|}{ Soclals } \\
\hline LAI & $53.933,000$ & $96.200,000$ & $89.860,000$ & $84.000,000$ & $53.440,000$ & $62.266,000$ & $97.000,000$ & $90.000,000$ & 82000,000 & $55.822,000$ & $68.931,000$ & $97.600,000$ & $90.200,000$ & $81.000,000$ & $59.000,000$ \\
\hline LA7 & 0,278 & 0,010 & 0,033 & 0,036 & 0,112 & 0,145 & - & 0,411 & 0,037 & 0,215 & 0,218 & 0,031 & 0,333 & 0,099 & 0,288 \\
\hline $\mathrm{L} 470$ & 0,970 & 0,110 & 0,900 & 0,390 & 0,510 & 0,770 & 0,480 & 1,800 & 0,370 & 0,340 & 0,760 & 0,480 & 1,500 & 0,320 & 0,350 \\
\hline
\end{tabular}

Table3 - Performance Matrix - 2008 - 2010

\begin{tabular}{|c|c|c|c|c|c|c|c|c|c|c|c|c|c|c|c|}
\hline \multirow[t]{3}{*}{ Cilterla } & \multicolumn{15}{|l|}{ Alternatives } \\
\hline & \multicolumn{5}{|l|}{2008} & \multicolumn{5}{|l|}{2009} & \multicolumn{5}{|l|}{2010} \\
\hline & E1 & E2 & E3 & $E 4$ & $E 5$ & E1 & E2 & E3 & E4 & $E 5$ & $\mathrm{E} 1$ & E2 & E3 & E4 & $E 5$ \\
\hline \multicolumn{16}{|l|}{ Econom/c } \\
\hline $\mathrm{BCl}$ & 2,400 & 3,838 & 1,580 & 3,900 & 2,590 & 1,791 & 3,998 & 1,590 & 3,900 & 2,630 & 2,120 & 3,000 & 1,996 & 4,060 & 2,602 \\
\hline $\mathrm{BCS}$ & 5,000 & 4,000 & 2,000 & 5,000 & 2,000 & 5,000 & 4,000 & 2,000 & 5,000 & 2,000 & 5,000 & 3,000 & 2,000 & 5,000 & 2,000 \\
\hline \multicolumn{16}{|c|}{ Environmentel } \\
\hline $\mathrm{EN} 3$ & 612,564 & 749,534 & 946,072 & 976,466 & $1.019,728$ & 806,494 & 712,685 & 943,913 & 1032,666 & $1.006,302$ & 707,170 & 700,847 & 846,502 & 825,787 & 999,624 \\
\hline EN8 & 222,808 & 242,706 & 388,417 & 211,451 & 284,551 & 269,230 & 239,846 & 341,173 & 209,343 & 277,098 & 240,940 & 202,412 & 656,732 & 181,787 & 296,721 \\
\hline EN16 & 65,605 & 438,299 & 130,050 & 92,027 & 43,582 & 94,888 & 44,543 & 115,48 & 89,919 & 41,981 & 69,892 & 133,950 & 134,499 & 92,210 & 56,312 \\
\hline Diso & 0,788 & 6,567 & 136,986 & 13,347 & 66,175 & 1,239 & 6,579 & 9,994 & 12,785 & 0,938 & 1,430 & 5,472 & 34,696 & $10.669,000$ & 16,961 \\
\hline $\mathbb{1} 20$ & 161,861 & 49,969 & 303,451 & 133,474 & 132,226 & 207,109 & 47,969 & 242,557 & 112,399 & 147,924 & 185,169 & 65,517 & 362,694 & 140,772 & 125,57 \\
\hline D 60 & 279,863 & 128,492 & 260,101 & 105,374 & 126,677 & 339,659 & 123,349 & 24,680 & 91,324 & 127,090 & 299,745 & 136,760 & 257,281 & 101,297 & 136,151 \\
\hline EN21 & 206,781 & 53,538 & 312,121 & 139,094 & 204,157 & 301,661 & 41,116 & 291,204 & 132,771 & 310,433 & 226,975 & 73,684 & 585,249 & 180,165 & 309,541 \\
\hline QN22 & 266,467 & 356,921 & 291,313 & 271,163 & 165,018 & 816,870 & 455,428 & $3.620,229$ & 573,235 & 176,051 & 509,985 & 204,904 & 1002,594 & 262,584 & 211,757 \\
\hline Q123 & 499,717 & 242,706 & 353,737 & 148,226 & 135,227 & 388,549 & 82,233 & 258,465 & 169,301 & 232,304 & 420,554 & 327,417 & 639,845 & 171,962 & 304,265 \\
\hline B 30 & $966.896,477$ & $2141.526,337$ & $5.548 .812,207$ & $3.652968,037$ & $1.586 .712,, 15$ & $1728.697,196$ & $1.701535,699$ & $4.480 .055,139$ & $3.582718,651$ & 2.083.41, 846 & 1.1.191.135, 458 & $2039.418,289$ & $5.842440,687$ & $2.802 .452,120$ & $1963.838,205$ \\
\hline \multicolumn{16}{|l|}{ Soclals } \\
\hline LAl & $74.240,000$ & 92000,000 & $90,390,000$ & $80.000,000$ & $60.000,000$ & $76.919,000$ & $80.300,000$ & $90.000,000$ & 81000,000 & $59.800,000$ & $67.257,800$ & $92.620,000$ & $90,090,000$ & $816.000,000$ & $57.624,400$ \\
\hline LA? & 0,242 & 0,022 & 0,022 & $\cdot$ & $\cdot$ & 0,091 & $\cdot$ & 0,011 & 0,049 & $\cdot$ & 0,195 & 0,013 & 0,162 & 0,044 & 0,12 \\
\hline LA70 & 0,592 & 0,430 & 0,600 & 0,360 & 0,360 & 0,480 & 0,340 & 0,400 & 0,300 & 0,270 & 0,714 & 0,368 & 1,040 & 0,348 & 0,366 \\
\hline
\end{tabular}

Table4 - Performance Matrix - 2011 - 2013

\begin{tabular}{|c|c|c|c|c|c|c|c|c|c|c|c|c|c|c|c|}
\hline \multirow[t]{3}{*}{ Cilterla } & \multicolumn{15}{|l|}{ Alternatives } \\
\hline & \multicolumn{5}{|l|}{2011} & \multicolumn{5}{|l|}{2012} & \multicolumn{5}{|l|}{2013} \\
\hline & $\mathrm{E} 1$ & $E 2$ & $E 3$ & $E 4$ & $E 5$ & E1 & $\mathrm{E} 2$ & E3 & $E 4$ & $E 5$ & E1 & $E 2$ & E3 & $E 4$ & $E 5$ \\
\hline \multicolumn{16}{|l|}{ Economlc } \\
\hline $\mathrm{BCl}$ & 1,956 & 3,499 & 1,793 & 3,980 & 2,616 & 2,136 & 3,565 & 1,897 & 3,555 & 2,789 & 2,257 & 3,456 & 1,901 & 3,495 & 2,888 \\
\hline$B C B$ & 5,000 & 3,000 & 2,000 & 5,000 & 2,000 & 5,000 & 3,000 & 3,000 & 3,000 & 1,000 & 5,000 & 3,000 & 3,000 & 3,000 & 4,000 \\
\hline \multicolumn{16}{|c|}{ Environmental } \\
\hline $\mathrm{EN} 3$ & 756,832 & 706,766 & 895,208 & 929,227 & $1.002,963$ & 658,696 & 707,569 & 898,569 & 989,633 & 999,365 & \begin{tabular}{ll|}
658,666 \\
\end{tabular} & 701,256 & 858,252 & 871,252 & 896,652 \\
\hline EN8 & 255,085 & 221,766 & 498,953 & 195,662 & 286,909 & 263,058 & 221,565 & 426,365 & 156,325 & 298,256 & 255,787 & 215,236 & 426,333 & 154,210 & 298,222 \\
\hline EN16 & 82,390 & 89,247 & 124,973 & 91,065 & 49,147 & 80,369 & 90,336 & 125,365 & 256,256 & 48,265 & 81,525 & 91,256 & 124,252 & 255,222 & 49,352 \\
\hline Ev50 & 1,334 & 6,025 & 2,345 & 11,727 & 8,449 & 1,666 & 5,999 & 18,256 & 10,256 & 7,598 & 1,887 & 5,878 & 17,266 & 9,897 & 7,984 \\
\hline EN20 & 196,139 & 56,743 & 302,825 & 126,586 & 136,751 & 185,236 & 56,222 & 256,326 & 125,365 & 135,265 & 138,569 & 55,421 & 256,333 & 121,356 & 134,569 \\
\hline Q160 & 319,702 & 130,055 & 250,980 & 96,311 & 131,620 & 369,789 & 131,056 & 245,362 & 98,365 & 131,333 & 355,244 & 138,256 & 246,365 & 90,52 & 132,254 \\
\hline EN21 & 264,318 & 57,400 & 438,226 & 156,464 & 309,987 & 256,333 & 58,400 & 422,365 & 154,253 & 380,226 & 254,211 & 56,421 & 422,635 & 148,222 & 381,325 \\
\hline EN22 & 663,428 & 325,166 & 2311,411 & 417,909 & 193,904 & 701,553 & 325,166 & $1.896,325$ & 148,257 & 199,256 & 702,444 & 324,151 & 1752,236 & 138,254 & 197,365 \\
\hline E123 & 404,551 & 204,825 & 449,155 & 172,132 & 268,284 & 431,565 & 205,898 & 458,256 & 444,258 & 258,265 & 399,568 & 201,525 & 455,233 & 321,582 & 259,635 \\
\hline EN30 & 1.459.916,327 & $1870.476,994$ & $5.161 .247,913$ & $3.192585,385$ & $2.023 .640,025$ & $1589.999,898$ & 1.984.253,231 & $3.254 .125,362$ & $3.895 .365,254$ & $1.999 .854,213$ & $1.600 .002,210$ & $1.825 .632,012$ & $3.000 .252,256$ & $2.999 .654,215$ & $2000.125,210$ \\
\hline \multicolumn{16}{|l|}{ Soclals } \\
\hline LAI & $72.088,400$ & $86.460,000$ & $90.045,000$ & $81.300,000$ & $58.712,200$ & $71.888,000$ & $89.000,000$ & $85.265,023$ & $80,456,000$ & $58.666,136$ & $71.979,000$ & $85.000,000$ & $84.522,000$ & $79.654,000$ & $57.695,000$ \\
\hline LA? & 0,143 & 0,006 & 0,087 & 0,047 & 0,062 & 0,144 & 0,056 & 0,098 & 0,048 & 0,005 & 0,148 & 0,058 & 0,101 & 0,065 & 0,010 \\
\hline LA70 & 0,597 & 0,354 & 0,720 & 0,324 & 0,318 & 0,456 & 0,663 & 0,895 & 0,289 & 0,698 & 0,510 & 0,701 & 0,390 & 0,125 & 0,879 \\
\hline
\end{tabular}


The MCDA technology and the Oil and gas industry: chronologic analysis

Table5 - Thresholds - 2005 - 2009

\begin{tabular}{|c|c|c|c|c|c|c|c|c|c|c|c|c|c|c|c|}
\hline \multirow[t]{3}{*}{ Criteria } & \multicolumn{15}{|l|}{ Thresholds } \\
\hline & \multicolumn{3}{|l|}{2005} & \multicolumn{3}{|l|}{2006} & \multicolumn{3}{|l|}{2007} & \multicolumn{3}{|l|}{2008} & \multicolumn{3}{|l|}{2009} \\
\hline & $q$ & p & $v$ & $q$ & p & $v$ & $q$ & p & $v$ & $q$ & $p$ & $v$ & $q$ & p & $v$ \\
\hline \multicolumn{16}{|l|}{ Economic } \\
\hline $\mathrm{BCl}$ & 0,181 & 0,361 & 0,433 & 0,243 & 0,486 & 0,584 & 0,183 & 0,367 & 0,440 & 0,199 & 0,398 & 0,478 & 0,27 & 0,454 & 0,091 \\
\hline $\mathrm{ECS}$ & 0,200 & 0,400 & 0,480 & 0,261 & 0,522 & 0,626 & 0,200 & 0,400 & 0,480 & \begin{tabular}{l|l|l|l|l|l} 
& \\
\end{tabular} & 0,607 & 0,728 & \begin{tabular}{l|l|}
0,303 \\
\end{tabular} & 0,007 & 0,121 \\
\hline \multicolumn{16}{|c|}{ Environmental } \\
\hline $\mathbb{E} 3$ & 51,672 & \begin{tabular}{|l|l|}
103,344 & \\
\end{tabular} & 124,012 & 25,783 & 38,675 & 61,880 & 72,451 & 98,234 & 175,882 & 34,628 & 69,256 & 83,107 & \begin{tabular}{|l|l|}
27,324 & \\
\end{tabular} & 54,647 & 65,577 \\
\hline EN8 & 28,451 & 56,901 & 68,281 & 73,053 & 109,580 & 175,328 & 70,906 & 143,599 & 170,175 & 14,365 & 28,729 & 34,475 & 9,833 & 19,667 & 23,600 \\
\hline EN16 & 3,286 & 6,572 & 11,830 & 11,267 & 16,901 & 40,562 & 9,540 & 20,807 & 34,344 & 32,442 & 64,883 & 116,790 & 6,515 & 13,030 & 23,453 \\
\hline EN50 & 1,060 & 2,120 & 2,544 & 0,551 & 1,426 & 2,281 & 0,787 & 1,738 & 1,889 & 11,551 & 23,103 & 27,723 & 1,050 & 2,099 & 2,519 \\
\hline $\mathrm{BN} 20$ & 11,062 & 22,124 & 26,549 & 39,262 & 58,893 & 94,230 & 32,638 & 71,901 & 78,332 & 18,464 & 36,928 & 4,313 & 15,400 & 30,801 & 36,961 \\
\hline EN60 & 13,364 & 26,728 & 32,073 & 23,48 & 35,173 & 56,276 & 17,733 & 41,182 & 42,560 & 16,569 & 33,139 & 39,766 & \begin{tabular}{|l|l|}
20,835 \\
\end{tabular} & 41,670 & 50,004 \\
\hline EN21 & 20,766 & 41,531 & 49,837 & 77,190 & 115,785 & 185,256 & 68,432 & 145,622 & 164,237 & 19,072 & 38,14 & 45,772 & 24,376 & 48,753 & 58,503 \\
\hline $\mathrm{EN} 22$ & 39,362 & 78,724 & 141,703 & 23,691 & 35,536 & 85,286 & 32,185 & 55,876 & 115,867 & 13,800 & 27,600 & 49,680 & 282,628 & 565,256 & $1.017,462$ \\
\hline $\mathrm{EN} 23$ & 13,119 & 26,238 & 31,485 & 82,242 & 123,363 & 197,380 & 80,882 & 163,124 & 194,118 & 26,051 & 52,102 & 62,522 & 22,671 & 45,343 & 54,411 \\
\hline EN30 & $306.736,017$ & $613.472,034$ & $1.840 .416,103$ & \begin{tabular}{|l|}
$172.823,663$ \\
\end{tabular} & $345.647,325$ & 1.036.941,976 & $169.364,366$ & 338728,732 & \begin{tabular}{|l|}
$1016.186,197$ \\
\end{tabular} & \begin{tabular}{|l|l|}
$184.033,401$ \\
\end{tabular} & $368.066,803$ & $1.104 .200,408$ & \begin{tabular}{|l|l|l|l}
$125.171,120$ \\
\end{tabular} & $250.342,240$ & $751.026,719$ \\
\hline \multicolumn{16}{|l|}{ Socials } \\
\hline LA1 & $4.072,748$ & $8.145,496$ & $9.774,596$ & $3.544,204$ & \begin{tabular}{|l|l|}
$7.088,407$ \\
\end{tabular} & $8.506,089$ & $3.124,397$ & $6.668,600$ & $7,498,552$ & \begin{tabular}{|l|l|}
$2.613,156$ \\
\end{tabular} & $5.226,312$ & $6.271,574$ & $2.213,399$ & \begin{tabular}{|l|l|}
$4.426,799$ \\
\end{tabular} & $5.312,159$ \\
\hline LA7 & 0,022 & 0,044 & 0,053 & 0,033 & 0,065 & 0,089 & 0,025 & 0,058 & 0,061 & 0,021 & 0,042 & 0,050 & 0,008 & 0,016 & 0,019 \\
\hline LA70 & 0,072 & 0,14 & 0,172 & 0,122 & 0,244 & 0,293 & 0,098 & 0,220 & 0,235 & 0,024 & 0,048 & 0,057 & 0,017 & 0,034 & 0,040 \\
\hline
\end{tabular}

Table6 - Thresholds - 2010 - 2013

\begin{tabular}{|c|c|c|c|c|c|c|c|c|c|c|c|c|}
\hline \multirow[t]{3}{*}{ Criteria } & \multicolumn{12}{|l|}{ Thresholds } \\
\hline & \multicolumn{3}{|l|}{2010} & \multicolumn{3}{|l|}{2011} & \multicolumn{3}{|l|}{2012} & \multicolumn{3}{|l|}{2013} \\
\hline & $q$ & $p$ & $v$ & $q$ & $p$ & $v$ & $q$ & $p$ & $v$ & $q$ & $p$ & $v$ \\
\hline \multicolumn{13}{|l|}{ Economic } \\
\hline $\mathrm{EC} 1$ & 0,207 & 0,413 & 0,405 & 0,207 & 0,413 & 0,284 & 0,211 & 0,425 & 0,202 & 0,201 & 0,398 & 0,256 \\
\hline ECS & 0,253 & 0,507 & 0,487 & 0,253 & 0,507 & 0,425 & 0,264 & 0,528 & 0,415 & 0,152 & 0,325 & 0,189 \\
\hline \multicolumn{13}{|c|}{ Environmental } \\
\hline EN3 & 42,372 & 72,831 & 102,092 & 42,372 & 72,831 & 74,342 & 34,628 & 98,234 & 124,012 & 51,672 & 69,256 & 175,882 \\
\hline ENS & 39,322 & 71,767 & 94,372 & 39,322 & 71,767 & 29,038 & 14,365 & 143,959 & 68,281 & 28,451 & 28,729 & 170,175 \\
\hline EN16 & 12,610 & 24,439 & 45,396 & 12,610 & 24,439 & 70,122 & 32,442 & 20,807 & 11,830 & 3,286 & 64,883 & 34,344 \\
\hline EN50 & 3,080 & 6,097 & 7,391 & 3,080 & 6,097 & 15,121 & 11,551 & 1,738 & 2,544 & 1,060 & 23,103 & 1,889 \\
\hline $\mathrm{EN} 20$ & 23,365 & 44,129 & 56,077 & 23,365 & 44,129 & 40,637 & 18,464 & 71,901 & 26,549 & 11,062 & 36,928 & 78,332 \\
\hline EN60 & 18,390 & 35,578 & 44,136 & 18,390 & 35,578 & 44,885 & 16,569 & 41,182 & 32,073 & 13,364 & 33,139 & 42,560 \\
\hline EN21 & 41,967 & 77,967 & 100,721 & \begin{tabular}{ll|}
41,967 \\
\end{tabular} & 7,967 & 52,138 & 19,072 & 145,622 & 49,837 & 20,766 & 38,144 & 164,237 \\
\hline EN22 & 78,333 & 152,598 & 282,000 & 78,333 & 152,598 & 533,571 & 13,800 & 55,876 & 141,703 & 39,362 & 27,600 & 115,867 \\
\hline $\mathrm{EN} 23$ & 44,993 & 82,034 & 107,983 & 44,993 & 82,034 & 58,467 & 26,051 & 163,124 & 31,485 & 13,119 & 52,102 & 194,118 \\
\hline EN30 & $191.625,713$ & $383.251,427$ & 1.1.149.754,281 & $191.625,713$ & $383.251,427$ & $927.613,563$ & $184.033,401$ & $338.728,732$ & $1.840 .416,103$ & $306.736,017$ & $368.066,803$ & $1.016 .186,197$ \\
\hline \multicolumn{13}{|l|}{ Socials } \\
\hline LA1 & $3.113,581$ & $6.311,123$ & $7.472,594$ & $3.113,581$ & $6.311,123$ & $5.791,866$ & $2.613,156$ & $6.668,600$ & $9.774,596$ & $4.072,748$ & $5.226,312$ & $7.498,552$ \\
\hline LA7 & 0,022 & 0,045 & 0,054 & 0,022 & 0,045 & 0,034 & 0,021 & 0,058 & 0,053 & 0,022 & 0,042 & 0,061 \\
\hline LA70 & 0,066 & 0,138 & 0,160 & 0,066 & 0,138 & 0,049 & 0,024 & 0,220 & 0,172 & 0,072 & 0,048 & 0,235 \\
\hline
\end{tabular}

\subsection{Distillation}

A graph can be drawn from the credibility matrix (Tables 7 and 8). Each alternative is connected with another one by two arrows, one in each direction indicating the credibility index. The graph for many alternatives is highly complex. An automated procedure named distillation, should be used to rank the alternatives. The name "distillation" was chosen by analogy to alchemists who distill mixtures of liquid to extract a magic ingredient. The algorithm to classify all alternatives can be divided into two pre-classifications. The first pre-classification is achieved with descending distillation by selecting the best ranked alternatives initially and ending with the worst. The best alternative is extracted from the whole set by applying very strict rules (Eq. (8)). In this subset, the best alternatives are selected by application of less restrictive rules (Eq.(10)), and the same rules previously used would bring a different result. The procedure continues with less restrictive rules and a lower number of alternatives (subsets). The procedure ends when it remains only one alternative or a group of alternatives that cannot be separated. The second distillation uses the same procedure, but in the original set of alternatives removed, at first, the best results from the distillation. Thus, a new subset is obtained in each distillation, which contains the best alternative. In each distillation, the alternative extracted will be ranked at an inferior position. As an alternative is connected with each other by two arrows, one in each direction, but not necessarily with symmetrical credibility index; a second pre-classification is constructed with ascendant distillation. In this case, the worst alternatives are first selected and the distillation ends with the assign of the best alternative. For distillation, it is necessary that an alternative a preferred to $b$ is defined as follows: the alternative a preferred to $\mathrm{b}$ if the degree of credibility that "A exceeds B" is superior to the threshold $\lambda_{2}$ and significantly higher than the degree credibility "B exceeds A" (Eq. (8)).

$\mathrm{S}(\mathrm{A} ; \mathrm{B})>\lambda_{2}$ and $\mathrm{S}(\mathrm{A} ; \mathrm{B})-\mathrm{S}(\mathrm{B} ; \mathrm{A})>\mathrm{s}\left(\lambda_{0}\right)$

Where $\lambda_{2}$ is the highest level of credibility, which is slightly below the cutoff $\lambda_{1}$, as follows:

$\lambda_{2}=\operatorname{Max}_{\{S(A, B) \leq \lambda 1\}} S(A, B) \forall\{A, B\} \in G(9)$

Where $\mathrm{G}$ is the set of alternatives. $\lambda_{1}$ is the next level:

$\lambda_{1}=\lambda_{0}-\mathrm{s}\left(\lambda_{0}\right)$ 
where $\lambda_{0}$ is the greatest degree of credibility in the respective credibility matrix:

$\lambda_{0}=\operatorname{Max}_{A, B \in G} S(A, B)(11)$

and $\mathrm{s}\left(\lambda_{0}\right)$ is the following threshold discrimination:

$\mathrm{s}\left(\lambda_{0}\right)=\alpha+\beta . \lambda_{0}(12)$

It is used a $\alpha=0.3$ and $b \beta=-0.15$ since both values are recommended by Roy (1985).

Table7 - Credibility Matrix - $2005-2009$

\begin{tabular}{|c|c|c|c|c|c|c|c|c|c|c|c|c|c|c|c|c|c|c|c|c|c|c|c|c|c|c|}
\hline & 2005 & & & & & 2006 & & & & & & 07 & & & & & & 2008 & & & & & 2009 & & & \\
\hline & E1 & $\mathrm{F}$ & & E5 & & E1 & E2 & & & & & E1 & E & & & & & $\mathrm{E} 1$ & $\mathrm{E} 2$ & & & & & & & \\
\hline & 0,00 & 0,0010 & $0,00 \mid 0$, & 0,00 & & & 0,00 & 0, & 0,0 & 0,00 & & & $0,00 \mid 0$, & 0, & 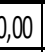 & & & & 0,00 & & 0,00 & El & & 0,00 & & 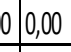 \\
\hline & 0,80 & 0,000 & $0,00 \mid 0$, & 0,9 & $\mathrm{E} 2$ & 0,93 & $t$ & 0,00 & 0,91 & 0,85 & E2 & 0,00 & 0 & 0,00 & 0,00 & 0,1 & E & 0,00 & [o, &, 000 & $\begin{array}{ll}00 & 0,00\end{array}$ & E2 & 0,88 & {$[0,0$} & $00 \mid 0,00$ & \\
\hline E3 & $0,00 \quad 0,00$ & & \begin{tabular}{l|l|l}
0,00 & 0,
\end{tabular} & 0,0 & E3 & 0,000 & 0,00 & & 0,00 & 0,00 & E3 & $0,00 \mathrm{c}$ & 0,00 & & 0,00 & 0,0 & E3 & & 0,00 & 0,0 & 0000,00 & $\mathrm{E} 3$ & & 0,00 & & 500 \\
\hline 24 & \begin{tabular}{|l|l|l|}
0,00 & 0,00 \\
\end{tabular} & 0,00 & $\begin{array}{l}-10,1 \\
\end{array}$ & 0,00 & E4 & $\overline{0,840} 0$ & $0,00 \mid 0$ & 0,00 & & 0,47 & E4 & 0,870 & \begin{tabular}{|l|l|}
0,74 & 0,1 \\
\end{tabular} & 0,00 &. & 0,86 & EA & 0,880, & 0,00 & \begin{tabular}{ll|}
, 93 & -
\end{tabular} & - 0,87 & EA & & & & 10 \\
\hline & \begin{tabular}{|l|l|}
75 & 0,00 \\
\end{tabular} & 0,000 & & & E5 & $\overline{0,000}$ & $0,00 \mid 0$ & 0,000 & 0,00 & & E5 & 0,000 & \begin{tabular}{l|l|l}
0,00 & 0,1 \\
\end{tabular} & & 0,00 & & 15 & & & \begin{tabular}{l|l}
, 00 & 0,00
\end{tabular} & & & & & \begin{tabular}{l|l}
00 & 0,00
\end{tabular} & \\
\hline
\end{tabular}

Table8 - Credibility Matrix - 2010 - 2013

\begin{tabular}{|c|c|c|c|c|c|c|c|c|c|c|c|c|c|c|c|c|c|c|c|c|c|c|c|}
\hline \multicolumn{6}{|c|}{2010} & \multicolumn{6}{|c|}{2011} & \multicolumn{6}{|c|}{2012} & \multicolumn{6}{|c|}{2013} \\
\hline & E1 & $\mathrm{E} 2$ & E3 & E4 & E5 & & E1 & E2 & E3 & E4 & E5 & & E1 & E2 & E3 & E4 & E5 & & $\mathrm{E} 1$ & E2 & E3 & E4 & E5 \\
\hline E1 & - & 0,00 & 0,00 & 0,00 & 0,00 & E1 & - & 0,00 & 0,00 & 0,00 & 0,00 & E1 & - & 0,00 & 0,00 & 0,00 & 0,00 & E1 & - & 0,00 & 0,00 & 0,00 & 0,00 \\
\hline E2 & 0,86 & - & 0,00 & 0,00 & 0,59 & E2 & 0,93 & - & 0,00 & 0,00 & 0,80 & E2 & 0,58 & - & 0,00 & 0,00 & 0,69 & E2 & 0,46 & - & 0,00 & 0,00 & 0,00 \\
\hline E3 & 0,00 & 0,00 & - & 0,00 & 0,00 & E3 & 0,00 & 0,00 & - & 0,00 & 0,00 & E3 & 0,00 & 0,00 & - & 0,00 & 0,70 & E3 & 0,00 & 0,00 & - & 0,00 & 0,63 \\
\hline $\mathrm{E} 4$ & 0,86 & 0,00 & 0,00 & - & 0,00 & E4 & 0,90 & 0,00 & 0,00 & - & 0,00 & E4 & 0,99 & 0,00 & 0,00 & - & 0,00 & E4 & 0,71 & 0,00 & 0,56 & - & 0,00 \\
\hline E5 & 0,76 & 0,00 & 0,00 & 0,00 & - & E5 & 0,81 & 0,00 & 0,00 & 0,00 & - & E5 & 0,78 & 0,00 & 0,00 & 0,00 & - & E5 & 0,66 & 0,00 & 0,00 & 0,00 & - \\
\hline
\end{tabular}

By applying this procedure for all from 2005 to 2013, there are the distillations shown in Figs. 4 and 5. It may be noted that the result of descendant distillation in 2006 was similar to that in 2005, the company E2 had preference over the others followed by the company E4. The others did not receive preferences related, resulting in indifference between them.

In the years 2007 and 2008, results of descendant distillation were similar; the company E4 had preference over the others. Indifference, in these two years, was among four other companies, highlighting the strong preference for the company E4.

The result of descendant distillation showed preference for the company E4, followed by the company E2 in 2009. Regarding the companies E1, E3 and E5, there was no preference between them. Finally, in 2010 and 2011 the resultwas similar, and the company E2 had preference over the others followed by the company E4 (Fig. 4).

The ascendant distillation showed that the company E1 got preference over the others, followed by companies E4 and E5 in 2006. The others did not receive preferences related, resulting in indifference between them.
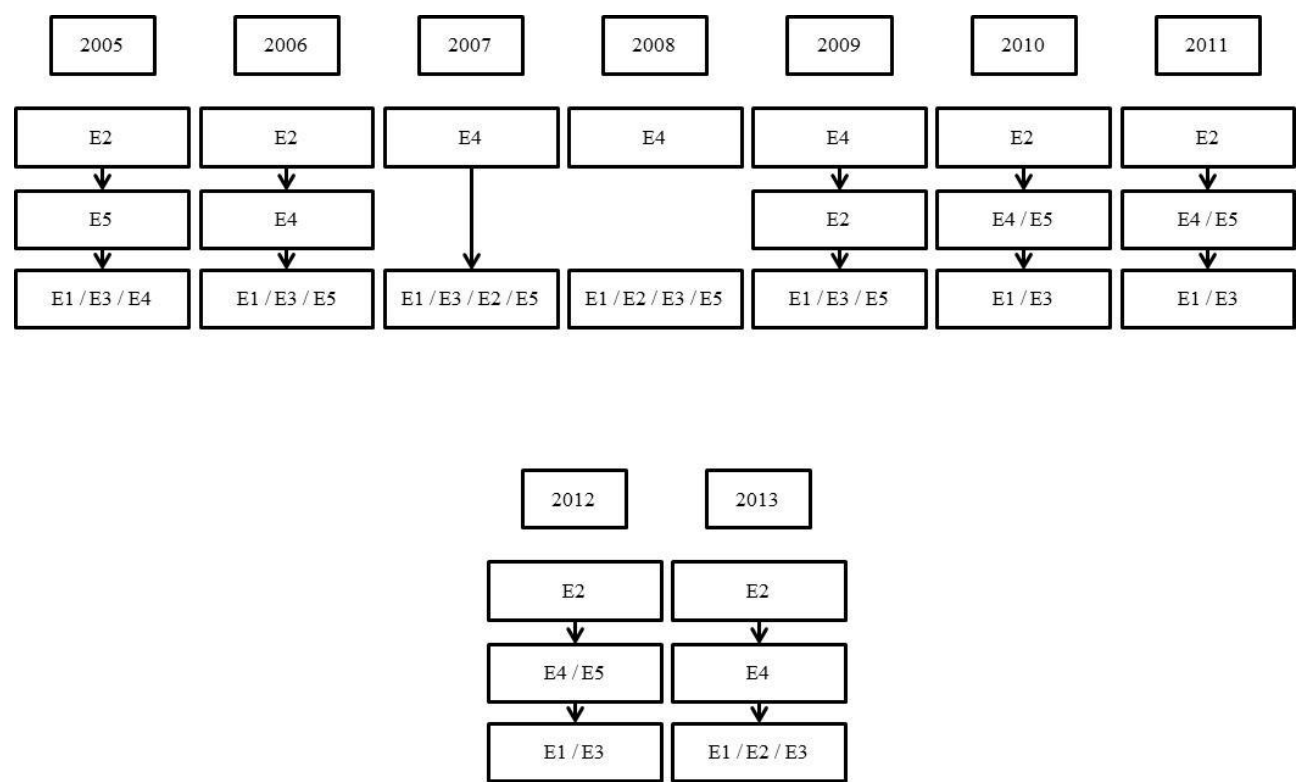

Fig.4 - Results from descendent distillations 
In the years 2007 and 2008, the results of ascendant distillation were similar. For the former, companies E1, E2 and E5 were ranked as the best and the companies at the second best position were E1, E3 and E5. It is noticed that only the E2 company is not indifferent to the other in the second year analyzed. For other companies, there was no preference between them. In 2009, the result of ascendant distillation showed preference for companies E1 and E5, followed by the company E3. Regarding the companies E2 and E5, there was no preference between them.

Finally, in 2010 and 2011 the result was similar, and the company E2 had preference over the others followed by the companies E4 and E5 (Fig. 5). In 2012 and 2013 the result was similar to 2010.
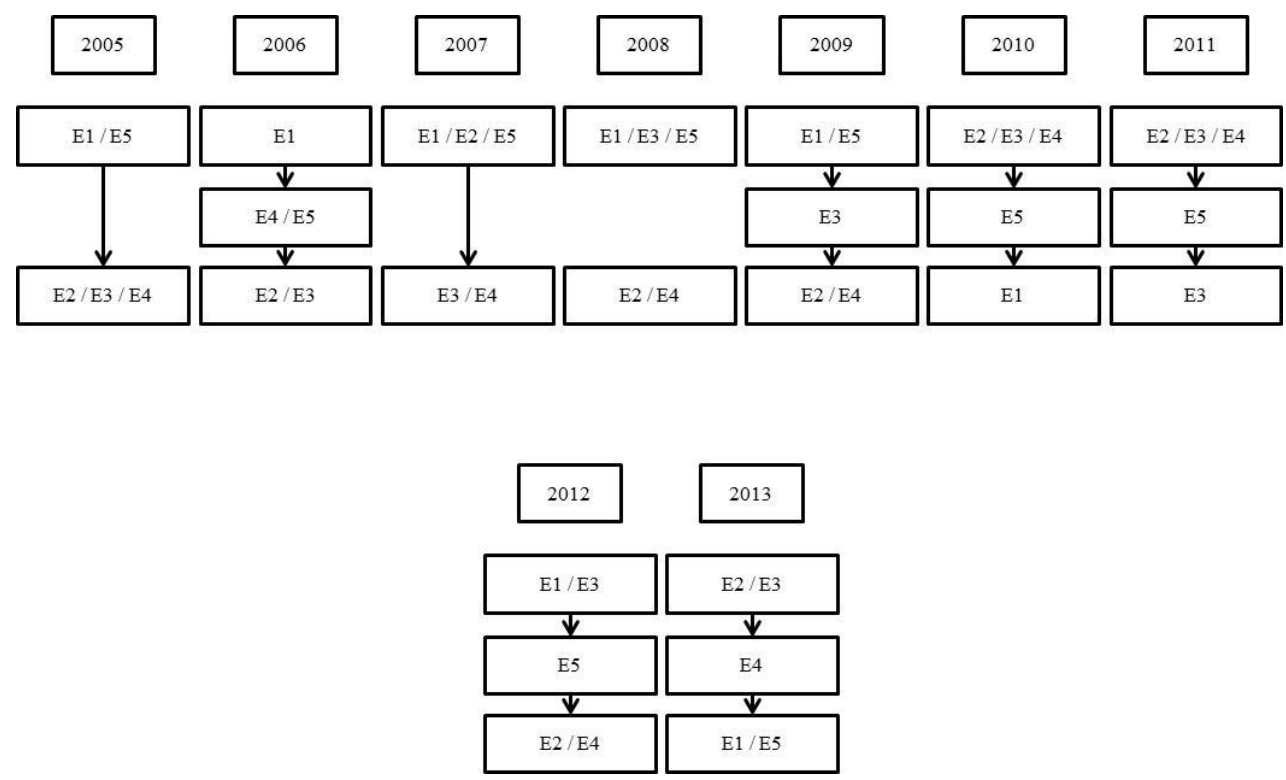

Fig.5 - Results from ascendent distillations

With successive distillations, the cutoff level $\lambda_{1}$ is gradually reduced, which makes it much easier to be preferred to B. However it contains some arbitrariness such as the recommended values of $\alpha$ and $\beta$ (Takeda, 2001). Other values may be used, which can slightly change the classification.

\subsection{Final Ordination}

The final ordination (Fig. 6) is obtained by combining two preclassifications. Refer to Section 4.2. Partial results of preclassifications are aggregated in the classification matrix. There are four possible cases $(\mathrm{Xu}$ and Ouenniche, 2012):

i. The alternative $\mathrm{A}$ is better than $\mathrm{B}$ or in both distillations or $\mathrm{A}$ is better than $\mathrm{B}$ in one distillation and it has the same position in the other one, subsequently $\mathrm{A}$ is better than $\mathrm{B}$ : $\mathbf{A} \mathbf{P}^{+} \mathbf{B}$;

ii. The alternative $\mathrm{A}$ is greater than $\mathrm{B}$ in one distillation, but $\mathrm{B}$ is better than $\mathrm{A}$ in another distillation, then $\mathrm{A}$ is incomparable to B: A R B;

iii. Alternative $\mathrm{A}$ has the same position that B in both distillations, therefore A is indifferent to B: A I B;

iv. $\mathrm{A}$ is smaller than $\mathrm{B}$ in both distillations or $\mathrm{A}$ is smaller than $\mathrm{B}$ in one distillation and it has the same rank in the other distillation, then $\mathrm{A}$ is worse than $\mathrm{B}: \mathrm{A} \mathbf{P}^{-} \mathbf{B}$.

The company E4 had the best performance, considering its evolution. This companywas indifferent to E3 (E4I E3) and the incomparable company E5 (E4 R E5) in 2005 and 2006, and it obtained the second position in the ordination; however, in the following years its performance was considered more relevant, enabling a prominent position before the others;

The company E2 obtained the second best performance, considering its evolution. The company ranked first in the ranking in 2005 and 2006, only falling to second position in the other years, except 2010 and 2011 , where E2 was first too. This favorable performance in seven years provided its effective implementation and criteria analyzed;

The company E3 has remained virtually constant in all years. In the years 2005 to 2007 it took the second position in the ranking, dropping to third in the years 2008 and 2009, which earned him third place overall. This company was considered indifferent to enterprises E1 and E5 (E1 I E3) and (E3 I E5) in 2008, which did not happen again in 2009. 
In 2010 and 2011, the company E2 had the best performance, considering this evolution. Not the same was considered in 2012 and 2013, where this company was the second.

The company E5 began at second position in the ordination in 2005, just indifferent to companies E2 and E4 (E4 I E5) and (E5 I E2). In the years 2006e2008 it remained at the third position, being indifferent to the companies E1 (E1 I E5), E2 (E5 I E2) and E3 (E3 I E5). In 2009 it got the last position, being indifferent to the company E1 only. In 2010 and 2011, this company had the third best performance, being indifferent by E3. This low performance improvement for company E5 allowed its fourth position overall in the final ordination. This similar result could be analyzed in 2012 and 2013.

The company E1 got the worse evolution according to the criteria analyzed. This got the last position every year, being indifferent to companies E2 (E1 I E2) and E5 (E5 I E1) in 2007,E3 (E1 I E3) and E5 (E5 I E1) in 2008, only the company E5 (E1 I E5) in 2009, and in 2010 to 2013 this companywas the worst.

In order to analyze the robustness of results, the sensitivity analysis was performed, whose weighted values, thresholds and criteria arrangements were varied.
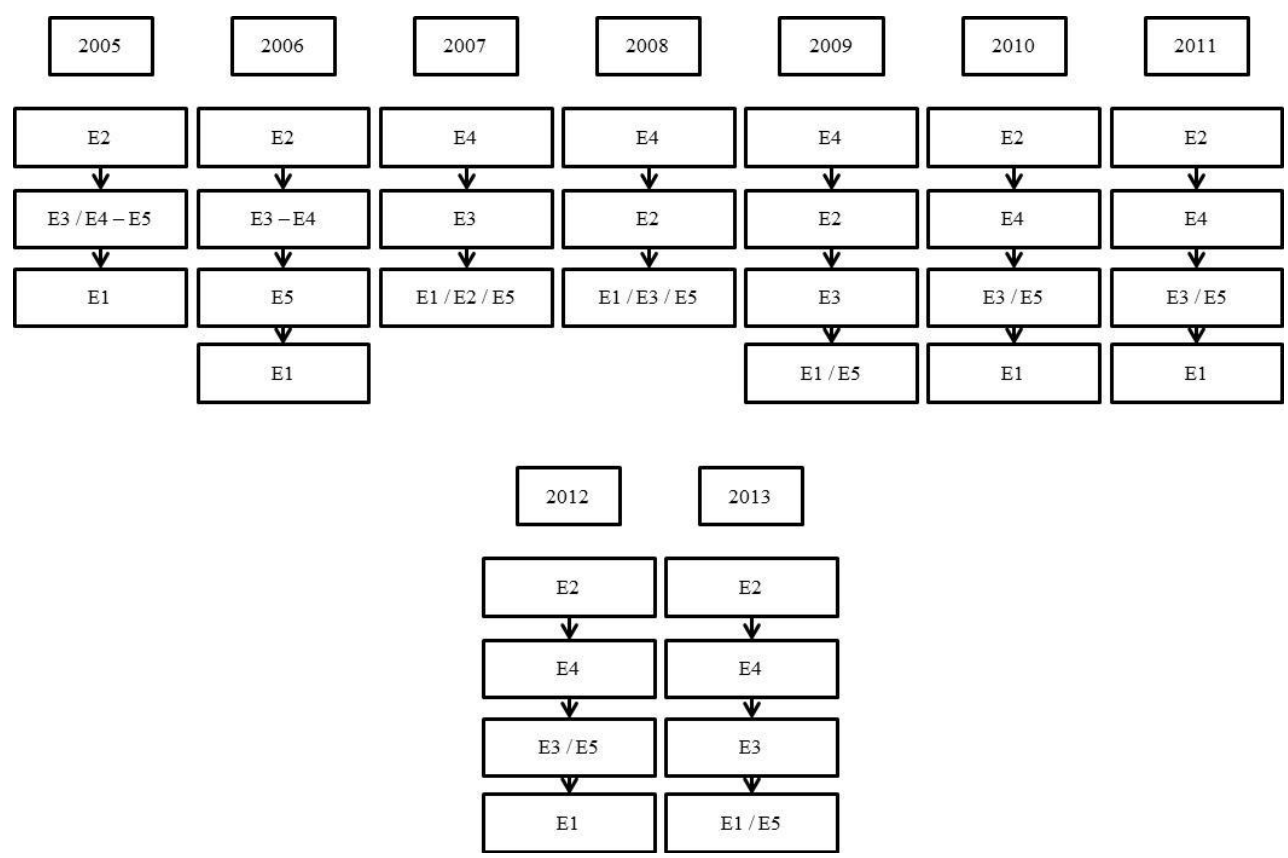

Fig.6 - Final Ordination

\subsubsection{Sensitivity analysis}

A sensitivity analysis (Tables 9 and 10) was carried out varying the weights and some criteria arrangements. This analysis was performed to obtain a greater robustness of the results. At the stage of new criteria, arrangements resulted in nine important combinations in order to verify the accuracy of the final ordination. The change in weights of the criteria groups, i.e. economic, social and environmental groups was performed by assigning weights between 1.5 and 2.5 to each group, resulting in six combinations. It is important to remember that the weights of all criteria were equal originally. A total of fifteen new combinations were performed to assess the final ordination's robustness, Fig. 6 . Tables 9 and 10 show the sensitivity analysis for the years 2005, 2006, 2007, 2008, 2009, 2010, 2011, 2012 and 2013. Checking for the sensitivity analyzes performed for each year surveyed (Tables 9 and 10), there is consistency in the results, which according to the final ordination has prevailed (Fig. 6). In 2005 the disparity in the new ordination after changes performed is negligible, as it can be seen in other years. The weights assigned confirmed that, even with the change in importance of the criteria groups, there is a big change in the ordination of companies, which features robustness to the final result. 
The MCDA technology and the Oil and gas industry: chronologic analysis

Table9 - SensibilityAnalysis - 2005 - 2009

\begin{tabular}{|c|c|c|c|c|c|c|c|c|c|c|c|c|c|c|}
\hline 2005 & & & 2006 & & & 2007 & & & 2008 & & & 2009 & & \\
\hline & Sensibility & Ranking & & Sensibility & Ranking & & Sensibility & Ranking & & Sensibility & Ranking & & Sensibility & Ranking \\
\hline 1 & $-(\mathbb{E N} 3)$ & $E 2-(E 3 ; E 4)\{[E 5]-E 1$ & 1 & $-(\mathbb{E N} 3)$ & E2-[E3][E4]-E5-E1 & 1 & $-(E \mathbb{E N} 3)$ & E4-E3-E2-(E1; $; 55)$ & 1 & $-(\mathbb{E N} 3)$ & $E 4-E 2-(E 1 ; E 3 ; E 5)$ & 1 & $-(E \mathbb{E N} 3)$ & $(E 2 ; E 4)-E 3-E 5-E 1$ \\
\hline 2 & -(EN3; EN16) & $\mathrm{E} 2-(\mathrm{E3} ; \mathrm{E} \mathrm{E})\{\mathrm{E} 5]-\mathrm{E} 1$ & 2 & -(EN3; EN16) & E2-[E3][E4]-E5-E1 & 2 & -(EN3; EN16) & E4-E3-(E1;E2; ;E5) & 2 & -(EN3; EN16) & E4-E2-E3-(E1;E5) & 2 & -(EN3; EN16) & E4-E2-E3-E5-E1 \\
\hline 3 & -(EN16) & $\mathrm{E} 2-(\mathrm{E3} ; \mathrm{E} 4)\{\mathrm{E} 5]-\mathrm{E} 1$ & 3 & -(EN16) & E2-[E3][E4]-E5-E1 & 3 & -(EN16) & E4-E3-(E1;E2; ;E5) & 3 & $-(E N 16)$ & E4-E2-E3-(E1;一E5) & 3 & -(EN16) & E4-E2-E3-(E1;:E5) \\
\hline 4 & -(EN21) & $\mathrm{E} 2-\mathrm{E} 4-[\mathrm{E} 3][\mathrm{E}]-\mathrm{E} 1$ & 4 & -(EN21) & E2-[E3][E4]-\{[E\}\}-E1 & 4 & -(EN21) & E4-E3-E2-(E1;E5) & 4 & -(EN21) & $\mathrm{E} 4 \mathrm{E} 2-(\mathrm{E} 1 ; \mathrm{E} ; \mathrm{F} ; \mathrm{E})$ & 4 & -(EN21) & E4-E2-E3-(E1;E5) \\
\hline 5 & -(EN21; EN22) & $E 2-E 4-[E 3][E 5]-E 2$ & 5 & -(EN21; EN22) & E2-[E3][E]\}-\{EE\}-E1 & 5 & -(EN21; EN22) & E4-E3-E2-(E1;E5) & 5 & -(EN21; EN22) & $E 4-E 2-(E 1 ; E 3 ; E 5)$ & 5 & -(EN21; EN22) & E4-E2-E3-(E1;:E5) \\
\hline 6 & -(EN22) & $\mathrm{E2}-(\mathrm{E} 3 ; \mathrm{E} 4)[\mathrm{E}] \mathrm{E}] \mathrm{E} 1$ & 6 & -(EN22) & E2-[E3][E4]-E5-E1 & 6 & -(EN22) & E4-E3-E2-(E1;一E) & 6 & -(EN22) & $E 4-E 2-(E 1 ; E 3 ; E 55)$ & 6 & -(EN22) & E4-E2-E3-(E1;E5) \\
\hline 7 & -(EN23) & E2-(E3;E4)[E5]-E2 & 7 & -(EN23) & E2-[E3][E4]-E5-E1 & 7 & $-(\mathrm{EN} 23)$ & E4-E3-E2-(E1;E5) & 7 & -(EN23) & $E 4-E 2-(E 1 ; E 3 ; ; E 5)$ & 7 & -(EN23) & E4-E2-E3-(E1;:E5) \\
\hline 8 & -(EN30) & E2-(E3;E4)[E5]-E3 & 8 & -(EN30) & E2-[E3][E4]-E5-E1 & 8 & -(EN30) & E4-E2-[E1][E5]-E3 & 8 & $-(\mathrm{EN} 30)$ & E4-[E1][E2]-E5-E3 & 8 & -(EN3O) & E2-E4-[E1][E5]-E3 \\
\hline 9 & $-(L A 7)$ & E2-(E3;E4)[E5]-E4 & 9 & $-(L A 7)$ & E2-[E3][E4]-E5-E1 & 9 & $-(L A 7)$ & E4-E3-(E1;E2; $; E 5)$ & 9 & $-(L A 7)$ & $E 4-E 2-(E 1 ; E 3 ; E 5)$ & 9 & $-(L A 7)$ & E4-E2-E3-(E1;:E5) \\
\hline & Weights & & & Weights & & & Weights & & & Weights & & & Weights & \\
\hline & Economics - 2 & & & Economics-2 & & & Economics - 2 & & & Economics-2 & & & Economics - 2 & \\
\hline 10 & & $\mathrm{E2}-(\mathrm{E} 3 ; \mathrm{E} 4) \mid[\mathrm{E}] \mathrm{E}]$ & 10 & & $\mathrm{E2}-[\mathrm{E}][\mathrm{E}-\mathrm{E}]-\mathrm{E} 5-\mathrm{E} 1$ & 10 & & E4-E3-(E1; $; 2 ; ; E 5)$ & 10 & & E4-E2-(E1;;E;;E5) & 10 & & E4-E2-E3-(E1;E5) \\
\hline & Environmental $-2,5$ & & & Environmental $-2,5$ & & & Environmental $-2,5$ & & & Environmental-2,5 & & & Environmental $-2,5$ & \\
\hline & Socials $-1,5$ & & & Socials $-1,5$ & & & Socials $-1,5$ & & & Socials $-1,5$ & & & Socials $-1,5$ & \\
\hline & Weights & & & Weights & & & Weights & & & Weights & & & Weights & \\
\hline & Economics - 2 & & & Economics - 2 & & & Economics - 2 & & & Economics - 2 & & & Economics - 2 & \\
\hline 11 & & $\mathrm{E2}-(\mathrm{E3} ; \mathrm{E} 4) \mid[\mathrm{E} 5]-\mathrm{E} 4$ & 11 & & $\mathrm{E2}-[\mathrm{E} 3][\mathrm{E} 4]-\mathrm{E} 5-\mathrm{E} 1$ & 11 & & E4-E3-E2-(E1;E5) & 11 & & $E 4-E 2-(E 1 ; E 3 ; ; E 5)$ & 11 & & E4-E2-E3-(E1;E5) \\
\hline & Environmental $-1,5$ & & & Environmental - 1,5 & & & Environmental $-1,5$ & & & Environmental-1,5 & & & Environmental $-1,5$ & \\
\hline & Socials $-2,5$ & & & Socials $-2,5$ & & & Socials $-2,5$ & & & Socials $-2,5$ & & & Socials $-2,5$ & \\
\hline & Weights & & & Weights & & & Weights & & & Weights & & & Weights & \\
\hline & Economics - 2,5 & & & Economics - 2,5 & & & Economics - 2,5 & & & Economics-2,5 & & & Economics - 2,5 & \\
\hline 12 & & $E 2-(E 3 ; E 4) \mid[E 5]-E 4$ & 12 & & $E 2-[E 3][E-4]-E 5-E 1$ & 12 & & E4-E3-(E1;E2;;E5) & 12 & & $E 4-E 2-(E 1 ; E 3 ; E E 5)$ & 12 & & E4-E2-E3-(E1;:E5) \\
\hline & Environmental-2 & & & Environmental - 2 & & & Environmental - 2 & & & Environmental-2 & & & Environmental - 2 & \\
\hline & Socials $-1,5$ & & & Socials $-1,5$ & & & Socials $-1,5$ & & & Socials $-1,5$ & & & Socials $-1,5$ & \\
\hline & Weights & & & Weights & & & Weights & & & Weights & & & Weights & \\
\hline & Economics - 2.5 & & & Economics - 2.5 & & & Economics - 2.5 & & & Economics - 2.5 & & & Economics - 2.5 & \\
\hline 13 & & $E 2-(E 3 ; E 4) \mid[E 5]-E 4$ & 13 & & $E 2-[E 3][E 4]-E 5-E 1$ & 13 & & $E 4-E 3-(E 1 ; E 2 ; E 5)$ & 13 & & E4-E2-(E1;E3;EE) & 13 & & E4-E2-E3-(E1;-E5) \\
\hline & Environmental - 1,5 & & & Environmental $-1,5$ & & & Environmental $-1,5$ & & & Environmental-1,5 & & & Environmental $-1,5$ & \\
\hline & Socials-2,5 & & & Socials $-2,5$ & & & Socials $-2,5$ & & & Socials $-2,5$ & & & Socials $-2,5$ & \\
\hline & Weights & & & Weights & & & Weights & & & Weights & & & Weights & \\
\hline & Economics $-1,5$ & & & Economics - 1,5 & & & Economics $-1,5$ & & & Economics - 1,5 & & & Economics - 1,5 & \\
\hline 14 & & $E 2-([3 ; E 4) \mid[E 5]-E 4$ & 14 & & E2-[E3][E4]-E5-E1 & 14 & & E4-E3-(E1;E2; $; 5)$ & 14 & & $E 4-E 2-([1 ; ;-33 ;[5])$ & 14 & & $(E 2 ; E 4)$-E3-E5-E1 \\
\hline & Environmental $-2,5$ & & & Environmental $-2,5$ & & & Environmental $-2,5$ & & & Environmental-2,5 & & & Environmental $-2,5$ & \\
\hline & Socials-2 & & & Socials-2 & & & Socials-2 & & & Socials-2 & & & Socials-2 & \\
\hline & Weights & & & Weights & & & Weights & & & Weights & & & Weights & \\
\hline & Economics - 1,5 & & & Economics - 1,5 & & & Economics - 1,5 & & & Economics - 1,5 & & & Economics - 1,5 & \\
\hline 15 & & $E 2-(E 3 ; E 4) \mid[E 5]-E 4$ & 15 & & $\mathrm{E2}-[\mathrm{E}][\mathrm{E}-\mathrm{E}]-\mathrm{E} 5-\mathrm{E} 1$ & 15 & & E4-E3-E2-(E1;EE) & 15 & & $E 4-E 2-(E 1 ; \in 3 ; ; E 5)$ & 15 & & $(E 2 ; E 4)-E 3-E 5-E 1$ \\
\hline & Environmental -2 & & & Environmental - 2 & & & Environmental -2 & & & Environmental-2 & & & Environmental -2 & \\
\hline & Socials $-2,5$ & & & Socials $-2,5$ & & & Socials $-2,5$ & & & Socials $-2,5$ & & & Socials $-2,5$ & \\
\hline
\end{tabular}


Table10 - SensibilityAnalysis - 2010 - 2013

\begin{tabular}{|c|c|c|c|c|c|c|c|c|c|c|c|}
\hline 2010 & & & 2011 & & & 2012 & & & 2013 & & \\
\hline & Sensibility & Ranking & & Sensibility & Ranking & & Sensibility & Ranking & & Sensibility & Ranking \\
\hline 1 & $-(E N 3)$ & E2-E4-[E3][E5];E1 & 1 & $-(\mathrm{EN} 3)$ & E2-E4-[E3][E5];E1 & 1 & $-(\mathrm{EN} 3)$ & E2-E4-[E3][E5];E1 & 1 & $-(\mathrm{EN} 3)$ & E2-E4-E3-(E1;E5) \\
\hline 2 & -(EN3; EN16) & E2-E4-[E3][E5];E1 & 2 & -(EN3; EN16) & E2-E4-[E3][E5];E1 & 2 & -(EN3; EN16) & E2-E4-[E3][E5];E1 & 2 & -(EN3; EN16) & E2-E4-E3-(E1;E5) \\
\hline 3 & -(EN16) & E2-E4-[E3][E5];E1 & 3 & $-($ EN16) & E2-E4-[E3][E5];E1 & 3 & -(EN16) & E2-E4-[E3][E5];E1 & 3 & $-($ EN16) & E2-E4-E3-(E1;E5) \\
\hline 4 & -(EN21) & E2-E4-[E3][E5];E1 & 4 & $-($ EN21) & E2-E4-[E3][E5];E1 & 4 & -(EN21) & E2-E4-[E3][E5];E1 & 4 & $-(E N 21)$ & E2-E4-E3-(E1;E5) \\
\hline 5 & -(EN21; EN22) & E2-E4-[E3][E5];E1 & 5 & -(EN21; EN22) & E2-E4-[E3][E5];E1 & 5 & -(EN21; EN22) & E2-E4-[E3][E5];E1 & 5 & -(EN21; EN22) & E2-E4-E3-(E1;E5) \\
\hline 6 & $-($ EN22) & E2-E4-[E3][E5];E1 & 6 & $-($ EN22) & E2-E4-[E3][E5];E1 & 6 & -(EN22) & E2-E4-[E3][E5];E1 & 6 & $-($ EN22) & E2-E4-E3-(E1;E5) \\
\hline 7 & $-($ EN23) & E2-[E3][E5]-E4-E1 & 7 & $-($ EN23) & E4-[E3][E5]-E2-E1 & 7 & -(EN23) & $\mathrm{E2}-\mathrm{E} 4-(\mathrm{E} 3 ; \mathrm{ES}) ; \mathrm{E} 1$ & 7 & $-($ EN23) & E2-E4-E3-E1-E5 \\
\hline 8 & -(EN30) & E2-E4-E5-E3-E1 & 8 & -(EN30) & E4-E2-E5-E3-E1 & 8 & -(EN30) & E2-E4-(E3;E5);E1 & 8 & $-($ EN30) & E2-E4-E3-E1-E5 \\
\hline 9 & $-($ LA7) & E2-[E3][E5]-E4-E1 & 9 & $-(L A 7)$ & E2-E4-[E3][E5];E1 & 9 & $-(L A 7)$ & E2-E4-(E3;E5);E1 & 9 & $-($ LA7) & E2-E4-E3-E1-E5 \\
\hline & Weights & & & Weights & & & Weights & & & Weights & \\
\hline & Economics - 2 & & & Economics - 2 & & & Economics - 2 & & & Economics - 2 & \\
\hline 10 & & E2-[E3][E5]-E4-E1 & 10 & & E4-E2-[E3][E5];E1 & 10 & & E2-E4-[E3][E5];E1 & 10 & & E2-E4-E3-(E1;E5) \\
\hline & Environmental - 2,5 & & & Environmental - 2,5 & & & Environmental - 2,5 & & & Environmental - 2,5 & \\
\hline & Socials $-1,5$ & & & Socials $-1,5$ & & & Socials - 1,5 & & & Socials $-1,5$ & \\
\hline & Weights & & & Weights & & & Weights & & & Weights & \\
\hline & Economics - 2 & & & Economics - 2 & & & Economics - 2 & & & Economics - 2 & \\
\hline 11 & & E2-[E3][E5]-E4-E1 & 11 & & E4-E2-[E3][E5];E1 & 11 & & E2-E4-[E3][E5];E1 & 11 & & E2-E4-E3-(E1;E5) \\
\hline & Environmental - 1,5 & & & Environmental - 1,5 & & & Environmental - 1,5 & & & Environmental - 1,5 & \\
\hline & Socials $-2,5$ & & & Socials - 2,5 & & & Socials - 2,5 & & & Socials - 2,5 & \\
\hline & Weights & & & Weights & & & Weights & & & Weights & \\
\hline & Economics - 2,5 & & & Economics - 2,5 & & & Economics - 2,5 & & & Economics - 2,5 & \\
\hline 12 & & E2-[E3][E5]-E4-E1 & 12 & & E2-E4-[E3][E5];E1 & 12 & & E2-E4-[E3][E5];E1 & 12 & & E2-E4-E3-(E1;E5) \\
\hline & Environmental - 2 & & & Environmental - 2 & & & Environmental - 2 & & & Environmental - 2 & \\
\hline & Socials $-1,5$ & & & Socials $-1,5$ & & & Socials - 1,5 & & & Socials - 1,5 & \\
\hline & Weights & & & Weights & & & Weights & & & Weights & \\
\hline & Economics - 2.5 & & & Economics - 2.5 & & & Economics - 2.5 & & & Economics - 2.5 & \\
\hline 13 & & E2-[E3][E5]-E4-E1 & 13 & & E2-E4-[E3][E5];E1 & 13 & & E2-E4-[E3][E5];E1 & 13 & & E2-E4-E3-(E1;E5) \\
\hline & Environmental - 1,5 & & & Environmental - 1,5 & & & Environmental - 1,5 & & & Environmental - 1,5 & \\
\hline & Socials - 2,5 & & & Socials $-2,5$ & & & Socials - 2,5 & & & Socials $-2,5$ & \\
\hline & Weights & & & Weights & & & Weights & & & Weights & \\
\hline & Economics - 1,5 & & & Economics - 1,5 & & & Economics - 1,5 & & & Economics - 1,5 & \\
\hline 14 & & E2-[E3][E5]-E4-E1 & 14 & & E2-E4-[E3][E5];E1 & 14 & & E2-E4-[E3][E5];E1 & 14 & & E2-E4-E3-(E1;E5) \\
\hline & Environmental - 2,5 & & & Environmental - 2,5 & & & Environmental - 2,5 & & & Environmental - 2,5 & \\
\hline & Socials - 2 & & & Socials - 2 & & & Socials - 2 & & & Socials - 2 & \\
\hline & Weights & & & Weights & & & Weights & & & Weights & \\
\hline & Economics - 1,5 & & & Economics - 1,5 & & & Economics - 1,5 & & & Economics - 1,5 & \\
\hline 15 & & E2-[E3][E5]-E4-E1 & 15 & & E2-E4-[E3][E5];E1 & 15 & & E2-E4-[E3][E5];E1 & 15 & & E2-E4-E3-(E1;E5) \\
\hline & Environmental - 2 & & & Environmental - 2 & & & Environmental - 2 & & & Environmental - 2 & \\
\hline & Socials - 2,5 & & & Socials - 2,5 & & & Socials - 2,5 & & & Socials - 2,5 & \\
\hline
\end{tabular}


It was observed that the criterion - EN30 e Total investments and operating costs - was significant in all years analyzed, since its withdrawal from the analysis directly impacted the finalordination, resulting in indifference between enterprises E1, E2and E5. In the years 2005 and 2006 the criterion of greatestimpact was EN 21 - Total water discharge by quality and destination - whose withdrawal from the analysis partially modifiedthe final ordination, causing incomparability of the E5 companyin relation to the others and indifference between companies E3and E4.

The variation of weights in the criteria groups had a major impact only in 2009, where amendments 15 and 16 partially modified the companies' final ordination, changing the indifference to companies E2 and E4, which was previously observed in companies E1 and E5.

\section{Conclusions}

The system application provided the ranking of companies, which proved to be little susceptible to the variation of criteria weights, as well as in changing the arrangement of some other criteria.

The application of the method ELECTRE III promoted working on the objective (criteria values) and subjective (weights and criteria thresholds) variables in combination, characteristic that directs a hierarchy process understood as more sensitive to the complexity of decisions.

The criteria presented and discussed were adequate for evaluating the companies in the oil and gas sector, as they encompassed economic, environmental and social aspects for the study. It should be noted that, regarding the risks to the environmental criteria, there is need for a more accurate survey in the field, in order to evaluate all parameters that influence such a criterion, but for the present study, the evaluation performed was satisfactory. The study allowed analyzing the companies, strategically, checking for their development and performance in the years studied. According to the criteria selected, these companies were ordered to obtain comparisons and improvements in their production processes.

\section{References}

[1]. Bana e Costa, C.A., Oliveira, M.D., 2012. A multicriteria decision analysis model for faculty evaluation. Omega 40,424 - 436.

[2]. Bottero, M., Comino, E.,Duriavig, M., Ferretti, V., Pomarico, S., 2013. The application of a Multicriteria Spatial Decision Support System (MCSDSS) for the assessment of biodiversity conservation in the Province of Varese (Italy). Land Use Policy 30,730 - 738.

[3]. Castellini, C., Boggia, A., Cortina, C., Dal Bosco, A., Novelli, E., Mugnai, C., 2012. A multicriteria approach for measuring the sustainability of different poultry production systems. Journal of Cleaner Production 37, 192 - 201.

[4]. Cavallaro, F., 2010. A comparative assessment of thin-film photovoltaic production processes using the ELECTRE III method. Energy Policy 38, 463 - 474.

[5]. Durbach, I.N., Stewart, T.J., 2012. A comparison of simplified value function approaches for treating uncertainty in multi-criteria decision analysis. Omega 40, $456-464$.

[6]. Frini, A., Guitouni, A., Martel, J.M., 2012. A general decomposition approach for multi-criteria decision trees. European Journal of Operational Research 220, 452 - 460.

[7]. Giannoulis, C., Ishizaka, A., 2010. A Web-based decision support system with ELECTRE III for a personalised ranking of British universities. Decision Support Systems 48, 488 - 497.

[8]. Giner-Santonja, G., Aragonés-Beltrán, P., Niclós-Ferragut, J., 2012. The application of the analytic network process to the assessment of best available techniques. Journal of Cleaner Production 25, 86 - 95.

[9]. Global Reporting Initiative, 2015 (accessed 11.02.15). www.globalreporting.org.

[10]. Hanandeh, A., El-Zein, A., 2010. The development and application of multicriteria decision-making tool with consideration of uncertainty: the selection of a management strategy for the bio-degradable fraction in the municipal solid waste. Bioresource Technology 101, 555 - 561.

[11]. Hart, S.L., 1996. Beyond Greening: Strategies for a Sustainable World. Harvard Business Review.

[12]. Infante, C.E.D.C., Mendonça, F. M., Purcidonio, P. M., Valle, R., 2013. Triple bottom line analysis of oil and gas industry with multicriteria decision making. Journal of Cleaner Production 52, $289-300$.

[13]. Karmperis, A.C., Aravossis, K., Tatsiopoulos, I.P., Sotirchos, A., 2013. Decision support models for solid waste management: review and game-theoretic approaches. Waste Management (accessed 25.02.15.). http://dx.doi.org/10.1016/j.wasman.2013.01.017.

[14]. Kumah, A., 2006. Sustainability and gold mining in the developing world. Journal of Cleaner Production 14, 315 - 323.

[15]. Lang, D.J., Binder, C.R., Scholz, R.W., Wiek, A., Stäubli, B., Sieber, C., 2007. Sustainability Potential Analysis (SPA) of landfills d a systemic approach: initial application towards a legal landfill assessment. Journal of Cleaner Production 15,1654 - 1661.

[16]. Li, H., Sun, J., 2010. Business failure prediction using hybrid2 case-based reasoning (H2CBR). Computers \& Operations Research $37,137-151$.

[17]. Loken, E., 2007. Use of multicriteria decision analysis methods for energy planning problems. Renewable and Sustainable Energy Reviews 11, 1584 - 1595.

[18]. Montazer, G.A., Saremi, H.Q., Ramezani, M., 2009. Design a new mixed expert decision aiding system using fuzzy ELECTRE III method for vendor selection. Expert Systems with Applications 36, 10837 - 10847.

[19]. Mushtaq Khan, M., Zaman, K., Irfan, D., Awan, U., Ali, G., Kyophilavong, P., Shahbaz, M., Naseem, I., 2015.Journal of Cleaner Production $\mathrm{xxx}, 1-11$.

[20]. Natividade-Jesus, E., Coutinho-Rodrigues, J., Antunes, C.H., 2007.A multicriteria decision support system for housing evaluation. Decision Support Systems 43, 779 - 790.

[21]. Passuello, A., Cadiach, O., Perez, Y., Schuhmacher, M., 2012. A spatial multicriteria decision making tool to define the best agricultural areas for sewage sludge amendment. Environment International 38, 1 - 9.

[22]. Roussat, N., Dujet, C., Méhu, J., 2009. Choosing a sustainable demolition waste management strategy using multicriteria decision analysis. Waste Management 29, 12 - 20.

[23]. Roy, B., 1985. Multicriteria Decision Analysis.Économica, Paris, France. Takeda, E., 2001. A method for multiple pseudo-criteria decision problems. Computers \& Operations Research 28, 1427 - 1439. 
[24]. Tosic, N., Marinkovic, T. D., Stanic, M., 2015. Multicriteria optimization of natural and recycled aggregate concrete for structural use. Journal of Cleaner Production 87, $766-776$.

[25]. Worrall, R., Neil, D., Brereton, D., Mulligan, D., 2009. Towards a sustainability criteria and indicators framework for legacy mine land. Journal of Cleaner Production 17, 1426 - 1434.

[26]. Xu, B., Ouenniche, J., 2012. Performance evaluation of competing forecasting models: a multidimensional framework based on MCDA. Expert Systems with Applications 39, 8312 - 8324. 